\title{
ON SEQUENTIALLY RETRACTIVE INDUCTIVE LIMITS
}

\section{ARMANDO GARCÍA}

Received 27 May 2002

\begin{abstract}
Every locally complete inductive limit of sequentially complete locally convex spaces, which satisfies Retakh's condition $(M)$ is regular, sequentially complete and sequentially retractive. A quasiconverse for this theorem and a criterion for sequential retractivity of inductive limits of webbed spaces are given.
\end{abstract}

2000 Mathematics Subject Classification: 46A13, 46A30.

1. Introduction. Throughout the paper, $\left\{\left(E_{n}, \tau_{n}\right)\right\}_{n}$ is an inductive sequence of locally convex spaces and $(E, \tau)=\operatorname{ind}\left(E_{n}, \tau_{n}\right)$ is its inductive limit. Recall that $E$ is regular if every bounded subset in $E$ is contained and bounded in one of the steps, and $E$ is sequentially retractive if every null sequence in $E$ converges to zero in some step. We say that $E$ satisfies the Retakh's condition $(M)$ if in every space $E_{n}$, there is an absolutely convex neighborhood of zero $U_{n}$ such that

(1) $U_{n} \subset U_{n+1}$ for every $n \in \mathbb{N}$;

(2) for every $n \in \mathbb{N}$, there is $m>n$ such that all the topologies of the locally convex spaces $E_{k}$, for $k \geq m$, coincide on $U_{n}$. Equivalently, $\tau$ and $\tau_{m}$ coincide on $U_{n}$.

We assume that every such $U_{n}$ is $\tau_{n}$-closed and that $\tau_{n+1}$ and $\tau$ induce the same topology on $U_{n}$, which we do without loss of generality.

Finally, we say that $E$ satisfies condition $(Q)$ (see [8]) if part (1) in $(M)$ is dropped.

Vogt in [7] studied condition ( $M$ ) for LF-spaces, that is, for inductive limits of metrizable and complete (equivalently and sequentially complete) locally convex spaces. He obtained several important results about them; for example, that on LF-spaces, condition $(M)$ implies completeness, regularity, and sequential retractivity. Recently, Wengenroth in [8] proved the following very important result on LF-spaces: condition $(M)$, condition $(Q)$, acyclicity and sequential retractivity are equivalent.

On the other hand, Gómez-Wulschner and Kučera in [2, 3] studied sequential completeness and weak regularity conditions for inductive limits of sequentially complete spaces. They have shown that a regular inductive limit of sequentially complete spaces is sequentially complete [3].

In Theorem 2.5 we show a result similar to Vogt's, but in the context of a locally complete inductive limit with condition $(M)$ of a sequence of sequentially complete locally convex spaces. 
The last part is devoted to webbed spaces (definitions are recalled in that section). We present a quasiconverse to Theorem 2.5 and a criterion for sequential retractivity.

2. Regularity and sequential retractivity. Recall that a disk $D$ in a locally convex space $F$ is an absolutely convex, bounded and closed subset. We write $\left(F_{D}, \rho_{D}\right)$ to denote a normed space, where $F_{D}=\operatorname{span} D$ and $\rho_{D}$ is the norm topology generated on $F_{D}$ by the Minkowski's functional of $D$; equivalently, $\rho_{D}$ is generated by the basis of neighborhoods $\{\lambda D: \lambda>0\}$. Note that closedness is not necessary for the Minkowski's functional to be a norm.

In order to obtain the first theorem, we need a technical lemma and a pair of useful propositions.

LEMMA 2.1. If $E=\operatorname{ind} E_{n}$ satisfies condition $(M)$ for the sequence $\left(U_{n}\right)_{n}$, then ${\overline{U_{j}}}^{E}=\bigcup_{k=j}^{\infty}{\overline{U_{j}}}^{E}$ for every $j \in \mathbb{N}$.

Proof. Since $\tau$ restricted to $E_{k}$ is coarser than $\tau_{k}$, we have ${\overline{U_{j}}}^{E_{k}} \subset{\overline{U_{j}}}^{E}$ for $k \geq j$. So, $\bigcup_{k=j}^{\infty}{\overline{U_{j}}}^{E_{k}} \subset{\overline{U_{j}}}^{E}$. Conversely, let $x \in{\overline{U_{j}}}^{E}$. There exists a net $\left(x_{\alpha}\right)_{\alpha} \subset$ $U_{j}$ such that $x_{\alpha} \stackrel{\tau}{\rightarrow} x$. This implies that there exists $n \geq j$ and $\lambda>0$ such that $\lambda\left\{\left(x_{\alpha}\right)_{\alpha}, x\right\} \subset U_{n}$. Since $\tau$ and $\tau_{n+1}$ coincide on $U_{n}, \lambda x_{\alpha} \stackrel{\tau_{n+1}}{\longrightarrow} \lambda x$; so, $x_{\alpha} \stackrel{\tau_{n+1}}{\longrightarrow} x$. Hence, $x \in{\overline{U_{j}}}^{E_{n+1}}$.

The next proposition is the key to Theorem 2.5.

Proposition 2.2. Let every $\left(E_{n}, \tau_{n}\right)$ be locally complete. If $E=\operatorname{ind} E_{n}$ satisfies condition $(M)$, then every Banach disk $B \subset E$ is contained and bounded in some $E_{n}$.

Proof. Let $B \subset E$ be a Banach disk. By [5, Proposition 8.5.20], there exists $p \in \mathbb{N}$ such that $B \subset p{\overline{U_{p}}}^{E}=p \bigcup_{k=p}^{\infty}{\overline{U_{p}}}^{E_{k}}$, the last identity follows from Lemma 2.1 .

Since $B$ is $\tau$-closed and $\tau$-bounded, $B \cap E_{k}$ is $\tau_{k}$-closed and $B \cap p{\overline{U_{p}}}^{E_{k}} \subset B$ is $\tau$-bounded, for every $k \geq p$. Let $B_{k}=B \cap p{\overline{U_{p}}}^{E_{k}}$. We assume that every $U_{k}$ is $\tau_{k}$-closed, then $(1 / p) B_{k} \subset{\overline{U_{p}}}^{E_{k}} \subset{\overline{U_{k}}}^{E_{k}}=U_{k}$ for every $k \geq p$. By condition $(M), \tau$ and $\tau_{k+1}$ coincide on $U_{k}$, then $(1 / p) B_{k}$ is $\tau_{k+1}$-bounded. Now, the local completeness of $E_{k+1}$ implies that ${\overline{B_{k}}}^{E_{k+1}}$ is a Banach disk in $E_{k+1}$, so $\left(E_{\overline{B_{k}}} E_{k+1}, \rho_{\overline{B_{k}}} E_{k+1}\right)$ is a Banach space continuously embedded in $\left(E_{k+1}, \tau_{k+1}\right)$.

Note that for every $k \geq p$,

$$
{\overline{B_{k}}}^{E_{k+1}}={\overline{B \cap p{\overline{U_{p}}}^{E}}}^{E_{k}} E_{k+1} \subset{\overline{B \cap p{\overline{U_{p}}}^{E_{k+1}}}}^{E_{k+2}}={\overline{B_{k+1}}}^{E} E_{k+2} .
$$

This implies that ${\overline{B_{k}}}^{E_{k+1}}$ is contained in ${\overline{B_{k+1}}}^{E_{k+2}} \cap E_{\bar{B}_{k}} E_{k+1}$; therefore $\left(E_{\bar{B}_{k}} E_{k+1}\right.$, $\left.\rho_{\overline{B_{k}}} E_{k+1}\right)$ is continuously embedded in $\left(E_{\overline{B_{k+1}}} E_{k+2}, \rho_{\overline{B_{k+1}}} E_{k+2}\right)$. 
It follows that ind $\left(E_{\overline{B_{k}}} E_{k+1}, \rho_{\overline{B_{k}}} E_{k+1}\right)$ is an LB-space. In order to finish the proof, we prove that this is a nonproper LB-space. In other words, we show that there exists $k_{0} \in \mathbb{N}$ such that $\left(E_{\overline{B_{k_{0}}}} E_{k_{0}+1}, \rho_{\overline{B_{k_{0}}}} E_{k_{0}+1}\right)=\left(E_{B}, \rho_{B}\right)$.

Since $B$ is $T$-closed and $B_{k} \subset B$, we have ${\overline{B_{k}}}^{E_{k+1}} \subset B$. And ${\overline{B_{k}}}^{E_{k+1}} \subset B \cap E_{\bar{B}_{k}} E_{k+1}$ which implies that the identity map $i_{k}:\left(E_{\overline{B_{k}}} E_{k+1}, \rho_{\overline{B_{k}}} E_{k+1}\right) \rightarrow\left(E_{B}, \rho_{B}\right)$ is continuous for every $k \geq p$.

On the other hand,

$$
B=B \cap p \bigcup_{k=p}^{\infty}{\overline{U_{p}}}^{E_{k}}=\bigcup_{k=p}^{\infty} B \cap p{\overline{U_{p}}}^{E_{k}}=\bigcup_{k=p}^{\infty} B_{k} \subset \bigcup_{k=p}^{\infty}{\overline{B_{k}}}^{E_{k+1}} \subset B .
$$

This means that $\operatorname{span}(B)=\bigcup_{k=p}^{\infty} \operatorname{span}\left({\overline{B_{k}}}^{E_{k+1}}\right)$. Therefore, the identity map

$$
i: \text { ind }\left(E_{\overline{B_{k}}} E_{k+1}, \rho_{\overline{B_{k}}} E_{k+1}\right) \longrightarrow\left(E_{B}, \rho_{B}\right)
$$

is continuous and onto. By the open mapping theorem (see [5, Theorem 8.4.11]), the inverse identity map

$$
j:\left(E_{B}, \rho_{B}\right) \longrightarrow \operatorname{ind}\left(E_{\overline{B_{k}}} E_{k+1}, \rho_{\overline{B_{k}}} E_{k+1}\right)
$$

is continuous. By Jarchow [4, Corollary 5.6.4], the space $\left(E_{B}, \rho_{B}\right)$ is continuously embedded in some $\left(E_{\overline{B_{k_{0}}}} E_{k_{0}+1}, \rho_{\overline{B_{k_{0}}}} E_{k_{0}+1}\right)$.

We conclude that $B$ is contained and bounded in $\left(E_{k_{0}+1}, \tau_{k_{0}+1}\right)$.

COROLLARY 2.3. Let every $\left(E_{n}, \tau_{n}\right)$ be locally complete. If $E=\operatorname{ind} E_{n}$ is locally complete and satisfies condition $(M)$, then $E$ is regular.

Proposition 2.4. Let every $\left(E_{n}, \tau_{n}\right)$ be sequentially complete. If $E=\operatorname{ind} E_{n}$ is regular and satisfies condition $(M)$ for a sequence $\left(U_{n}\right)_{n}$, then $E$ is sequentially complete and sequentially retractive.

Proof. Let $\left(x_{l}\right)_{l}$ be a Cauchy sequence in $(E, \tau)$. Then, $A=\left\{x_{l}: l \in \mathbb{N}\right\}$ is a $\tau$-bounded set. So, there exists $n \in \mathbb{N}$, such that $A \subset E_{n}$ and $A$ is $\tau_{n}$-bounded. There exists $s>0$ such that $s A \subset U_{n}$. Since $\tau$ and $\tau_{n+1}$ coincide on $U_{n}$, it follows that $\left(s x_{l}\right)_{l}$ is $\tau_{n+1}$-Cauchy, then $\tau_{n+1}$-convergent to $s x_{0}$, for some $x_{0} \in E_{n+1}$, hence $\left(x_{l}\right)_{l}$ is convergent to $x_{0}$ in $(E, \tau)$.

In an analogous way, it is straightforward to show that $(E, \tau)$ is sequentially retractive.

From the preceding results we conclude the following theorem.

THEOREM 2.5. Let every $\left(E_{n}, \tau_{n}\right)$ be sequentially complete. If $E=\operatorname{ind} E_{n}$ is locally complete and satisfies condition $(M)$, then $E$ is regular, sequentially complete, and sequentially retractive. 
3. Sequential retractivity on webbed spaces. We give now two results on sequential retractivity for certain webbed spaces. For convenience, we recall some basic facts about webs which we need. For more information about the basic properties of webs, we refer the reader to the works of De Wilde [9], Jarchow [4], and Robertson [6].

A strand of a web $\mathbb{W}$ on a locally convex space $(F, \tau)$ is a collection of members of $\mathbb{W}$, one from each layer, with the $(k+1)$ th member of the strand contained in the $k$ th member. Strands will be denoted by $\left(W_{k}\right)_{k}$. A web on $F$ is compatible with $\tau$ if for each neighborhood of zero $U$ in $(F, \tau)$ and for each strand $\left(W_{k}\right)_{k}$ of $\mathbb{W}$, there is $k_{0}$ such that $W_{k_{0}} \subset U$.

Following the idea of Wengenroth in the proof of [8, Proposition 2.3], we get a quasiconverse for Theorem 2.5. To simplify the notation in this proposition, we use $W_{k} \in \mathbb{W}$ to denote $W_{k_{1}, k_{2}, \ldots, k_{r}} \in \mathbb{W}$, that is, write only one index as for the elements of a specific strand.

Proposition 3.1. Let every $\left(E_{n}, \tau_{n}\right)$ be a webbed space and $E=\operatorname{ind} E_{n}$ sequentially retractive. Then, for every $N \in \mathbb{N}$ there is $n \geq N$ and an element $W_{k}^{N}$ of the web $\mathbb{W}^{(N)}$ on $E_{N}$, for some $k=k(N)$, such that $\tau$ and $\tau_{n}$ coincide on $W_{k}^{N}$.

Proof. Suppose that this proposition is not true. So, there exists $E_{n_{0}}$ such that for every element of its web, say $W_{k}^{n_{0}} \in \mathbb{W}^{n_{0}}$, and for each $N \in \mathbb{N}$ there exists $n>N$ such that $\tau_{n}$ restricted to $W_{k}^{n_{0}}$ is strictly coarser than $\tau_{N}$ restricted to $W_{k}^{n_{0}}$.

For such $n_{0}$, fix an element of the web $W_{k_{0}}^{n_{0}} \in \mathbb{W}^{n_{0}}$. Let $N=n_{0}$, then there exists $n_{1}>n_{0}$ such that $\tau_{n_{1}}$ restricted to $W_{k_{0}}^{n_{0}}$ is strictly coarser than $\tau_{n_{0}}$. So, there is a sequence $\left(x_{l}^{k_{0}}\right)_{l} \subset W_{k_{0}}^{n_{0}}$, which is $\tau_{n_{1}}$-null but not $\tau_{n_{0}}$-null. Find an element of the $\left(k_{0}+1\right)$ th layer of the web $W_{k_{0}+1}^{n_{0}} \in \mathbb{W}^{n_{0}}$, such that $W_{k_{0}+1}^{n_{0}}+$ $W_{k_{0}+1}^{n_{0}} \subset W_{k_{0}}^{n_{0}}$, and $n_{2}>n_{1}$ such that $\tau_{n_{2}}$ restricted to $W_{k_{0}+1}^{n_{0}}$ is strictly coarser than $\tau_{n_{1}}$ restricted to $W_{k_{0}+1}^{n_{0}}$. Then, there is a sequence $\left(x_{l}^{k_{0}+1}\right)_{l} \subset W_{k_{0}+1}^{n_{0}}$, which is $\tau_{n_{2}}$-null but not $\tau_{n_{1}}$-null. In this way, determine a strand $\left(W_{k_{0}+k}^{n_{0}}\right)_{k}$ of the web $\mathbb{W}^{n_{0}}$, an increasing sequence of natural numbers $\left(n_{k}\right)_{k}$, and a collection of sequences $\left[\left(x_{l}^{k_{0}+k}\right)_{l}\right]_{k}$ such that every $\left(x_{l}^{k_{0}+k}\right)_{l} \subset W_{k_{0}+k}^{n_{0}}$ is $\tau_{n_{k+1}}$-null but not $\tau_{n_{k}}$-null.

Let $U$ be a neighborhood of zero in $(E, \tau)$. Then, $U \cap E_{n_{0}}$ is a neighborhood of zero in $\left(E_{n_{0}}, \tau_{n_{0}}\right)$, so there exists $K \in \mathbb{N}$ such that $W_{k_{0}+k}^{n_{0}} \subset U \cap E_{n_{0}} \subset U$ if $k>K$. It implies that $\left(x_{l}^{k_{0}+k}\right)_{l} \subset U$ for every $l \in \mathbb{N}$ if $k>K$. Now, if $k \leq K$, then $\left(x_{l}^{k_{0}+k}\right)_{l}$ is a $\tau$-null sequence, since it is a $\tau_{n_{k+1}}$-null sequence. Hence, arranging the double-indexed sequence in any way into a single indexed sequence, it results a $\tau$-null sequence. So, this sequence should be convergent in some $E_{m}$ since $E$ is sequentially retractive. But this is not possible, since the sequence is not convergent in any $E_{m}$. Hence the proposition is true.

Recall that a space $F$ is strictly barreled if given any ordered web in $F$, there is a strand $\left(W_{k}\right)_{k}$ such that for every $k \in \mathbb{N}, \overline{W_{k}}$ is a neighborhood of zero. So, 
if in Proposition 3.1 everyone of the corresponding elements from the webs, where the topologies coincide is $\tau_{n}$-neighborhoods of zero, then $E$ satisfies condition $(Q)$, and hence by [8, Proposition 2.5], $E$ satisfies condition ( $M$ ).

Following Gilsdorf [1], a locally convex space $F$ is sequentially webbed if it has a compatible web $W$ such that for every null sequence $\left(x_{m}\right)_{m}$ in $(F, \tau)$, there exist a strand $\left(W_{k}\right)_{k}$ and a natural number $M_{k}$ for every $k \in \mathbb{N}$, such that $x_{m} \in W_{k}$ for all $m \geq M_{k}$. To simplify, we denote this condition by (\#).

From [4, Corollary 5.3.3(b)], the inductive limit $E=\operatorname{ind} E_{n}$ of a numerable sequence of webbed spaces is again webbed and admits a completing web $W$ such that $\mathbb{W}(n)=E_{n}$ for every $n \in \mathbb{N}$. Moreover, the $k$ th layer of the web $\mathbb{W}$ on $E$ is the collection of members of the $k$ th layer in the spaces $E_{n}$. In the next theorem, we use such a web on $E=$ ind $E_{n}$ in order to characterize sequential retractivity for inductive limits of sequentially webbed spaces.

THEOREM 3.2. Let every $\left(E_{n}, \tau_{n}\right)$ be a sequentially webbed space. $E=\operatorname{ind} E_{n}$ is sequentially retractive if and only if $E$ is sequentially webbed.

Proof. Suppose that $E$ is sequentially retractive. For any null sequence $\left(x_{m}\right)_{m}$ in $(E, \tau)$, there exists $n \in \mathbb{N}$ such that $\left(x_{m}\right)_{m}$ is a null sequence in $\left(E_{n}, \tau_{n}\right)$. So, there is a strand $\left(W_{k}^{(n)}\right)_{k}$ of the web $W^{(n)}$ on $E_{n}$ satisfying (\#) on $E_{n}$. Note that by the form of the web on $E,\left(W_{k}^{(n)}\right)_{k}$ is also a strand for the web $W$ on $E$. So, $E$ is sequentially webbed. Conversely, let $\left(x_{m}\right)_{m}$ be a null sequence in $(E, \tau)$; since $E$ is sequentially webbed, there is a strand $\left(W_{k}\right)_{k}$ of $\mathbb{W}$ on $E$ satisfying (\#). By the form of the web on $E, W_{1}=\mathbb{W}(n)=E_{n}$ for some $n \in \mathbb{N}$. So, this strand is contained in $E_{n}$ and it is a strand of $\mathbb{W}^{(n)}$ on $E_{n}$. Now, since $W^{(n)}$ is compatible with $\tau_{n}$, for every $U$-neighborhood of zero in $\left(E_{n}, \tau_{n}\right)$, there exists $k \in \mathbb{N}$ such that $W_{k} \subset U$. Hence, $x_{m} \in U$ for all $m \geq M_{k}$.

ACKNOWLEDGments. The author is very grateful to the "Centre de Recerca Matemática," Barcelona, Spain for the kind hospitality during the preparation of part of this paper, and also to the referee for many valuable comments.

\section{REFERENCES}

[1] T. E. Gilsdorf, The Mackey convergence condition for spaces with webs, Int. J. Math. Math. Sci. 11 (1988), no. 3, 473-483.

[2] C. Gómez-Wulschner and J. Kučera, Sequentially complete inductive limits and regularity, preprint.

[3] _ Sequential completeness of inductive limits, Int. J. Math. Math. Sci. 24 (2000), no. $6,419-421$.

[4] H. Jarchow, Locally Convex Spaces, B. G. Teubner, Stuttgart, 1981.

[5] P. Pérez Carreras and J. Bonet, Barrelled Locally Convex Spaces, North-Holland Mathematics Studies, vol. 131, North-Holland Publishing, Amsterdam, 1987.

[6] W. Robertson, On the closed graph theorem and spaces with webs, Proc. London Math. Soc. (3) 24 (1972), 692-738. 
[7] D. Vogt, Regularity properties of (LF)-spaces, Progress in Functional Analysis (Peñíscola, 1990), North-Holland Math. Stud., vol. 170, North-Holland Publishing, Amsterdam, 1992, pp. 57-84.

[8] J. Wengenroth, Acyclic inductive spectra of Fréchet spaces, Studia Math. 120 (1996), no. 3, 247-258.

[9] M. De Wilde, Closed Graph Theorems and Webbed Spaces, Research Notes in Mathematics, vol. 19, Pitman, Massachusetts, 1978.

Armando García: Instituto de Matemáticas, UNAM, Zona de la Investigación Científica, Circuito Exterior, Ciudad Universitaria, México, DF 04510, Mexico

E-mail address: agarcia@matem.unam.mx 


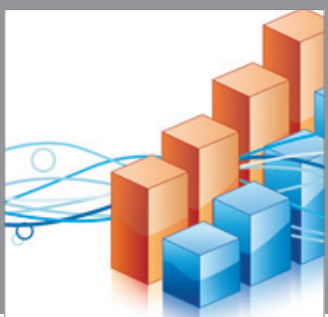

Advances in

Operations Research

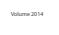

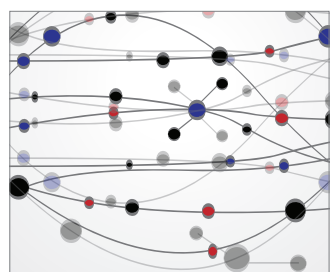

\section{The Scientific} World Journal
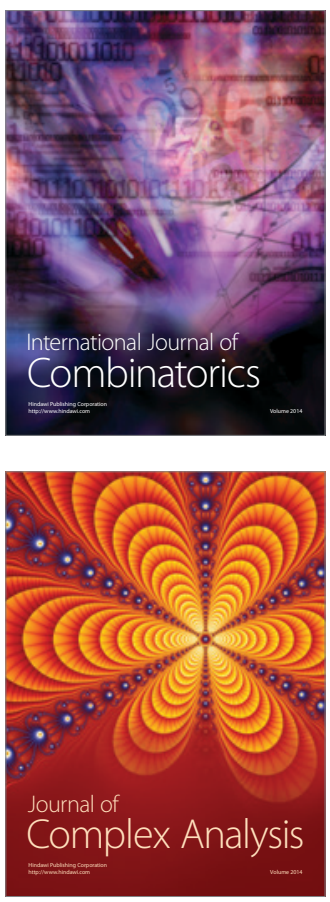

International Journal of

Mathematics and

Mathematical

Sciences
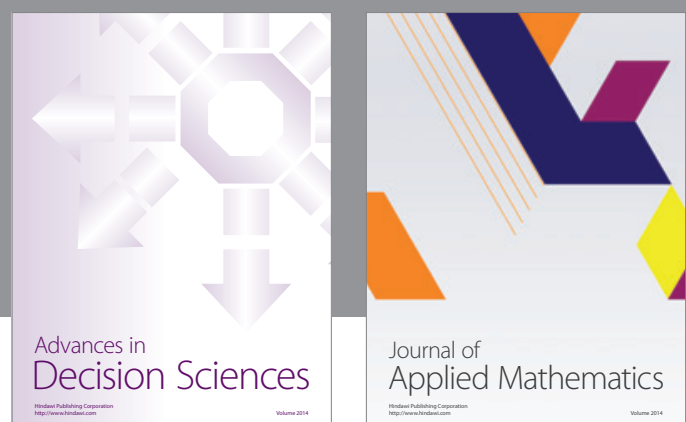

Journal of

Applied Mathematics
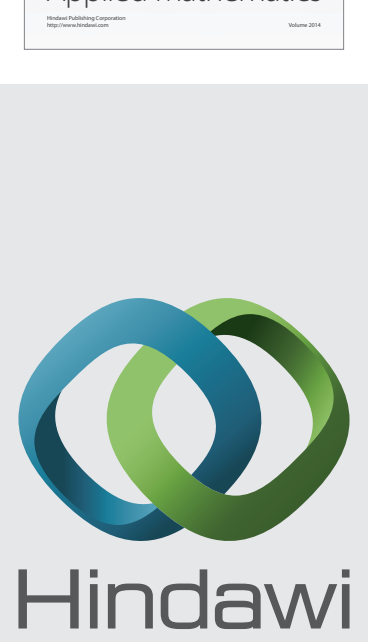

Submit your manuscripts at http://www.hindawi.com
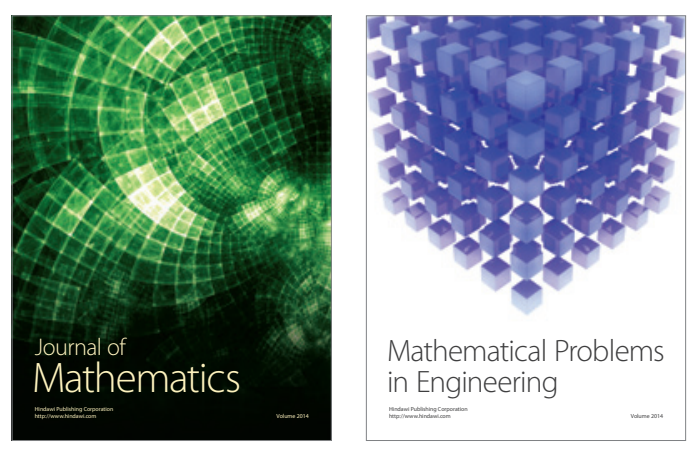

Mathematical Problems in Engineering
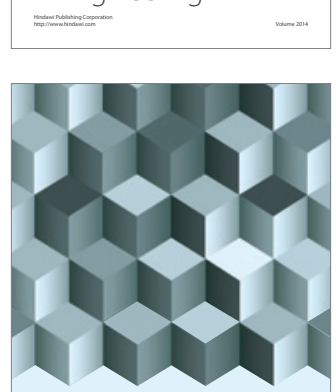

Journal of

Function Spaces
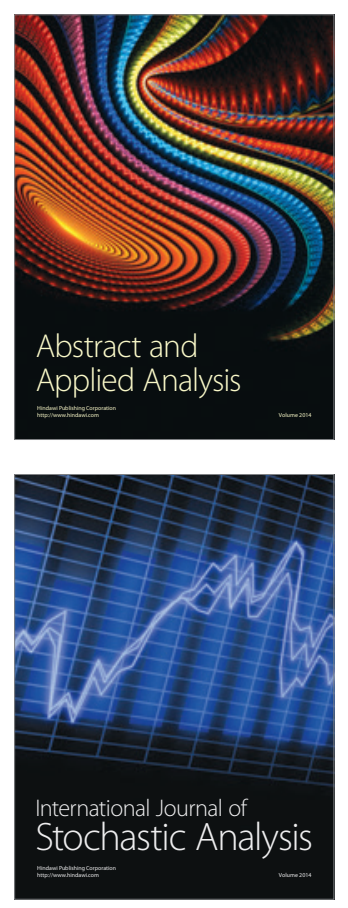

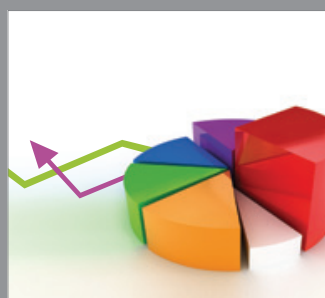

ournal of

Probability and Statistics

Promensencen
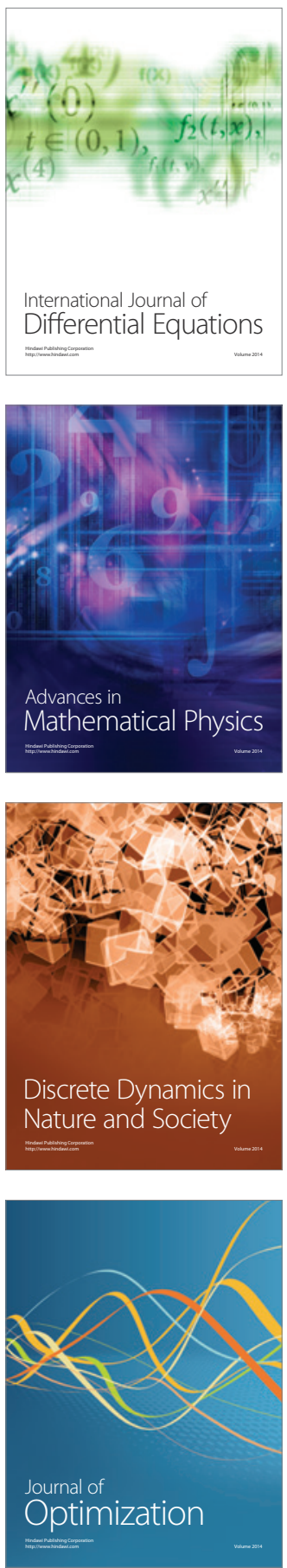